\title{
Unveiling shocks in planetary nebulae ${ }^{\star}$
}

\author{
Martín A. Guerrero ${ }^{1}$, Jesús A. Toalá ${ }^{1}$, Juan J. Medina ${ }^{1}$, Valentina Luridiana ${ }^{2,3}$, Luis F. Miranda ${ }^{4,5}$, \\ Angels Riera ${ }^{6,7}$, and Pablo F. Velázquez ${ }^{8}$ \\ ${ }^{1}$ Instituto de Astrofísica de Andalucía, IAA-CSIC, Glorieta de la Astronomía s/n, 18008 Granada, Spain \\ e-mail: mar@iaa.es \\ 2 Instituto de Astrofísica de Canarias, IAC, Vía Láctea s/n, 38205 La Laguna, Spain \\ 3 Departamento de Astrofísica, Universidad de La Laguna, 38200 La Laguna, Tenerife, Spain \\ ${ }^{4}$ Departamento de Física Aplicada, Facultade de Ciencias, Campus Lagoas-Marcosende s/n, Universidade de Vigo, 36310 Vigo, \\ Spain \\ 5 Consejo Superior de Investigaciones Científicas (CSIC), c/ Serrano 117, 28006 Madrid, Spain \\ 6 Departament de Física i Enginyeria Nuclear, EUETIB, Universitat Politècnica de Catalunya, Comte d’Urgell 187, 08036 Barcelona, \\ Spain \\ 7 Departament d'Astronomia i Meteorologia, Universitat de Barcelona, Av. Diagonal 647, 08028 Barcelona, Spain \\ 8 Instituto de Ciencias Nucleares, UNAM, Ciudad Universitaria, 04510 Mexico City, Mexico
}

Received 26 April 2013 / Accepted 25 July 2013

\begin{abstract}
The propagation of a shock wave into a medium is expected to heat the material beyond the shock, producing noticeable effects in intensity line ratios such as $[\mathrm{O} \mathrm{III}] / \mathrm{H} \alpha$. To investigate the occurrence of shocks in planetary nebulae (PNe), we have used all narrowband [O III] and $\mathrm{H} \alpha$ images of PNe available in the HST archive to build their [O III]/H $\alpha$ ratio maps and to search for regions where this ratio is enhanced. Regions with enhanced $[\mathrm{O}$ III] $/ \mathrm{H} \alpha$ emission ratio can be ascribed to two different types of morphological structures: bow-shock structures produced by fast collimated outflows and thin skins enveloping expanding nebular shells. Both collimated outflows and expanding shells are therefore confirmed to generate shocks in PNe. We also find regions with depressed values of the $[\mathrm{O} \mathrm{III}] / \mathrm{H} \alpha$ ratio which are found mostly around density bounded PNe, where the local contribution of [N II] emission into the F656N $\mathrm{H} \alpha$ filter cannot be neglected.
\end{abstract}

Key words. ISM: jets and outflows - ISM: general - planetary nebulae: general

\section{Introduction}

The increase in the local pressure of the material in a region makes it to expand, producing a disturbance in the surroundings that may drive a shock front. Shocks are ubiquitous in the universe whenever supernova explosions, photoionized gas, stellar winds, and collimated outflows drive shock waves in the circumstellar or interstellar media.

Shocks of fast moving outflows with material in the surrounding medium should produce appreciable effects. The most obvious is probably the formation of a bow-shock structure, as those associated with jet-like outflows in Herbig-Haro objects such as the archetypical HH34 and HH 111 (Reipurth et al. 1997, 2002; Raga et al. 2002). Planetary nebulae (PNe) also exhibit fast collimated outflows whose occurrence is found at very early stages of the nebular formation, including the late stages of the asymptotic giant branch (AGB) and the proto-PN phases. These collimated outflows can produce notable bowshock features, as those detected in IC 4593 (Corradi et al. 1997a).

On microscopic scales, a shock wave is an irreversible process, where entropy is generated as ordered kinetic energy is dissipated into heat. Therefore, a fast outflow propagating into

\footnotetext{
* Tables 1 and 2 are available in electronic form at
} http: //WwW . aanda.org a low density medium drives a forward shock which produces an increase of the electron temperature, $T_{\mathrm{e}}$. The increase in temperature would enhance the emission in the [O III] $\lambda 5007 \AA$ line, which is very sensitive to $T_{\mathrm{e}}$, whereas the low density would reduce the emissivity of the $\mathrm{H} \alpha$ line, which is more sensitive to the electron density, $N_{\mathrm{e}}$. Consequently, it is expected a region in front of the bow-shock at the tip of a collimated outflow where the $[\mathrm{O} \mathrm{III}] / \mathrm{H} \alpha$ ratio is significantly enhanced. Such caps of enhanced $[\mathrm{O} \mathrm{III}] / \mathrm{H} \alpha$ ratio have been revealed by HST images of IC 4634, a PN characterized by the presence of fast collimated outflows resulting in a point-symmetric morphology and clear bow-shock structures (Guerrero et al. 2008).

A dense, fast shell expanding into a tenuous, static medium can also propagate shocks into the surroundings. This effect is observed in bubbles blown by the wind of massive stars, such as S 308, the wind-blown bubble around the Wolf-Rayet star HD 50896, whose shell generates a forward shock that produces a notable offset between the [O III] and $\mathrm{H} \alpha$ emissions (Gruendl et al. 2000). Another example is provided by NGC 7635, a.k.a. the Bubble Nebula, a bubble blown by the wind of the massive O6.5 IIIf star BD $+60^{\circ} 2522$, where the offset between these emissions is $\approx 3 \times 10^{15} \mathrm{~cm}$ (Moore et al. 2002). The expansion of the different shells of PNe is also expected to generate shocks: one at the leading edge of the outer shell propagating into the unperturbed AGB wind and another at the leading 
edge of the bright rim expanding into the outer shell (e.g., Perinotto et al. 2004). Balick (2004) found a thin skin of enhanced $[\mathrm{O}$ III] $/ \mathrm{H} \alpha$ enveloping NGC 6543 in HST images of this nebula. The origin of this skin of enhanced [O III] $/ \mathrm{H} \alpha$ remained uncertain, but an observational artefact could be ruled out.

Fast collimated outflows are acknowledged to play an essential role in the nebular shaping in early stages of the PN formation (Sahai \& Trauger 1998). In particular, the expansion of bow-shock features has drawn the attention as a mechanism for the formation of bipolar and multi-polar PNe (see the review by Balick \& Frank 2002). Similarly, the expansion of the different nebular shells is critical for the evolution of PNe (Villaver et al. 2002; Perinotto et al. 2004). Both the shocks produced by fast collimated outflows and those produced by the shell expansion may have important dynamical effects in PNe, contributing decisively to the nebular shaping, evolution, and excitation.

Inspired by the results obtained for IC 4634 and NGC 6543, we have used the HST [O III] and $\mathrm{H} \alpha$ images of PNe available in the Mikulski Archive for Space Telescopes (MAST) to investigate the occurrence of skins of bright $[\mathrm{O}$ III $] / \mathrm{H} \alpha$ in $\mathrm{PNe}$ and its relationship with fast collimated outflows and expanding shells. The description of the search and analysis of HST images is presented in Sect. 2. Attending to their [O III]/H $\alpha$ ratio maps (Sect. 3), the PNe in this sample can be divided in four different types of which two of them show regions of enhanced $[\mathrm{O} \mathrm{III}] / \mathrm{H} \alpha$ emission associated with bow-shocks of collimated outflows and with expanding nebular shells, respectively. The results are further discussed in Sect. 4 and a short summary is given in Sect. 5.

\section{2. $\mathrm{H} \alpha$ and [O III] images of PNe in the HST archive}

We searched MAST for HST WFPC2 or WFC3 coeval $\mathrm{H} \alpha$ and [O III] images of PNe available by March 2013. This search yielded $\mathrm{H} \alpha$ and [O III] images for 103 PNe obtained through the F656N and F502N filters, respectively (Table 1). Since the adjacent [N II] $\lambda \lambda 6548,6584 \AA$ emission lines can be included in the bandpass of the $\mathrm{F} 656 \mathrm{~N} \mathrm{H} \alpha$ narrow-band filter, the potential contamination of the emission from these low-excitation lines on the $\mathrm{H} \alpha$ image needs to be discussed into further detail.

The bandpass of the WFC3 F656N H $\alpha$ filter only includes the contribution from the [N II] $\lambda 6548 \AA$ emission line with a filter transmission at its wavelength which is $\sim 5 \%$ that at the wavelength of the targeted $\mathrm{H} \alpha$ line (O'Dell et al. 2013). As for the WFPC2 F656N H $\alpha$ filter, its bandpass is broader (Lim et al. 2010) and includes contributions of both [N II] lines, with filter transmissions relative to that at the wavelength of the $\mathrm{H} \alpha$ line of $\sim 33 \%$ for the [N II] $\lambda 6548 \AA$ component and $\sim 5 \%$ for the [N II] $\lambda 6584 \AA$ line. Therefore, we can expect a $10 \%$ contamination by $[\mathrm{N}$ II] emission in the WFC 3 F656N filter when the [N II] $\lambda 6584 \AA$ line is 6 times brighter than the $\mathrm{H} \alpha$ line, but only $\sim 2 / 3$ as bright for the WFPC2 F656N filter.

The $[\mathrm{N}$ II] to $\mathrm{H} \alpha$ line ratio may be large for low-excitation PNe with bright [N II] emission lines. To preserve our sample from sources whose $\mathrm{H} \alpha$ image may be significantly contaminated by the contribution of $[\mathrm{N} \mathrm{II}]$ emission, hereafter we will exclude from our analysis those objects observed by the WFPC2 instrument with a [N II] $\lambda 6584 \AA$ to $\mathrm{H} \alpha$ line ratio greater than unity. This includes H 1-9, H 2-15, Hb 5, IC 4406, K 4-55, M 2-14, Mz 3, NGC 2438, NGC 2440, NGC 2818, NGC 3132, and NGC 6537. The source NGC 6302, observed with the WFC3 instrument, will be further excluded because its extremely high [N II] $\lambda 6584 \AA$ to $\mathrm{H} \alpha$ line ratio. The [O III]/H $\alpha$ ratio maps of sources with a [N II] $\lambda 6584 \AA$ to $\mathrm{H} \alpha$ line ratio close to unity will be carefully scrutinized to check for features associated to [N II] contamination. We note that the $(\mathrm{H} \alpha+[\mathrm{N} \mathrm{II}]) /[\mathrm{O}$ III $]$ ratio maps of PNe notably enhance the [N II] emission of low-ionization small-scale structures (Corradi et al. 1996). These features are easy to identify.

The images were downloaded from the HST archive and reduced using the standard pipeline procedure. For each PN, individual frames of the same epoch were combined to remove the cosmic rays using $\mathrm{IRAF}^{1}$ routines. The resulting $\mathrm{H} \alpha$ and [O III] images were subsequently used to generate [O III] to $\mathrm{H} \alpha$ ratio maps. Before producing these ratios, we checked the relative alignment of the images in the different filters. The WFC3 images were notably well aligned, but we noticed a consistent shift of $0 .{ }^{\prime} 1-0$. ' 3 between the WFPC2 $\mathrm{H} \alpha$ and [O III] images caused most likely by the different light path across the filters. Accordingly, the images in the different filters were aligned using stars in the field of view (including the central stars when available) and registered on the same grid. The accuracy of this procedure was usually better than $10 \%$ of a pixel, i.e., $\lesssim 0$ ' $^{\prime} 005$.

The $[\mathrm{O} \mathrm{III}] / \mathrm{H} \alpha$ ratio maps were examined to investigate the relative positions of the $[\mathrm{O}$ III] and $\mathrm{H} \alpha$ emissions and to search for regions of enhanced values of the $[\mathrm{O} \mathrm{III}] / \mathrm{H} \alpha$ ratio. The images of 18 PNe were found not suitable for this analysis: NGC 6853 (the Dumbbell Nebula) and NGC 7293 (the Helix Nebula) are much larger than the WFPC2 field of view, SaSt 1-1 and Th 4-1 are point sources, Hen 2-436, NGC 6833, and $\mathrm{PC} 11$ are compact PNe whose emission is not properly resolved, and H 1-43, H 1-55, H 2-25, H 2-26, Hen 2-262, JaFu 2, K 3-76, KFL 7, Th 3-6, Th 3-14, and Wray 16-282 are weak in [O III], resulting in poor quality $[\mathrm{O} \mathrm{III}] / \mathrm{H} \alpha$ ratio maps. All these $18 \mathrm{PNe}$ have been discarded from subsequent analysis.

\section{PN classification based on the $[\mathrm{O} I 1] / \mathrm{H} \alpha$ ratio}

The remaining $72 \mathrm{PNe}$ in our sample present $[\mathrm{O} \mathrm{III}] / \mathrm{H} \alpha$ ratio maps suitable for its analysis. The $\mathrm{H} \alpha$ and $[\mathrm{O}$ III] $/ \mathrm{H} \alpha$ ratio images of a representative number of PNe with adequate $[\mathrm{O}$ III] $/ \mathrm{H} \alpha$ ratio maps are presented in Figs. 1 through 4 . Greyscales in the $[\mathrm{O} \mathrm{III}] / \mathrm{H} \alpha$ ratio have been chosen to highlight the locations where the [O III] and $\mathrm{H} \alpha$ emissions show spatial differences. A close inspection of the $\mathrm{H} \alpha$ and $[\mathrm{O}$ III] $/ \mathrm{H} \alpha$ ratio images of these PNe has allowed us to classify them into four different types:

\section{- Type A}

PNe that present caps of enhanced $[\mathrm{O} \mathrm{III}] / \mathrm{H} \alpha$ ratios at the tip of jet-like features. These regions of bright [O III] emission can be interpreted as bow-shock features produced by fast collimated outflows. The $\mathrm{H} \alpha$ images and [O III] $/ \mathrm{H} \alpha$ ratio maps of the type A PNe IC 4593, IC 4634, NGC 3918, NGC 6210, NGC 6543, NGC 6572, and NGC 7009 are shown in Fig. 1.

\section{- Type B}

PNe surrounded by skins of enhanced [O III]/H $\alpha$ emission associated with nebular shells. In some double-shell PNe, both shells exhibit a skin of enhanced $[\mathrm{O}$ III $] / \mathrm{H} \alpha$. The list of type B PNe includes NGC 1501, NGC 2371-2, NGC 3242, NGC 6153, NGC 6818, NGC 6826, NGC 7009,

1 IRAF is distributed by the National Optical Astronomy Observatories which is operated by the Association of Universities for Research in Astronomy, Inc. (AURA) under cooperative agreement with the National Science Foundation. 
Martín A. Guerrero et al.: Unveiling shocks in PNe
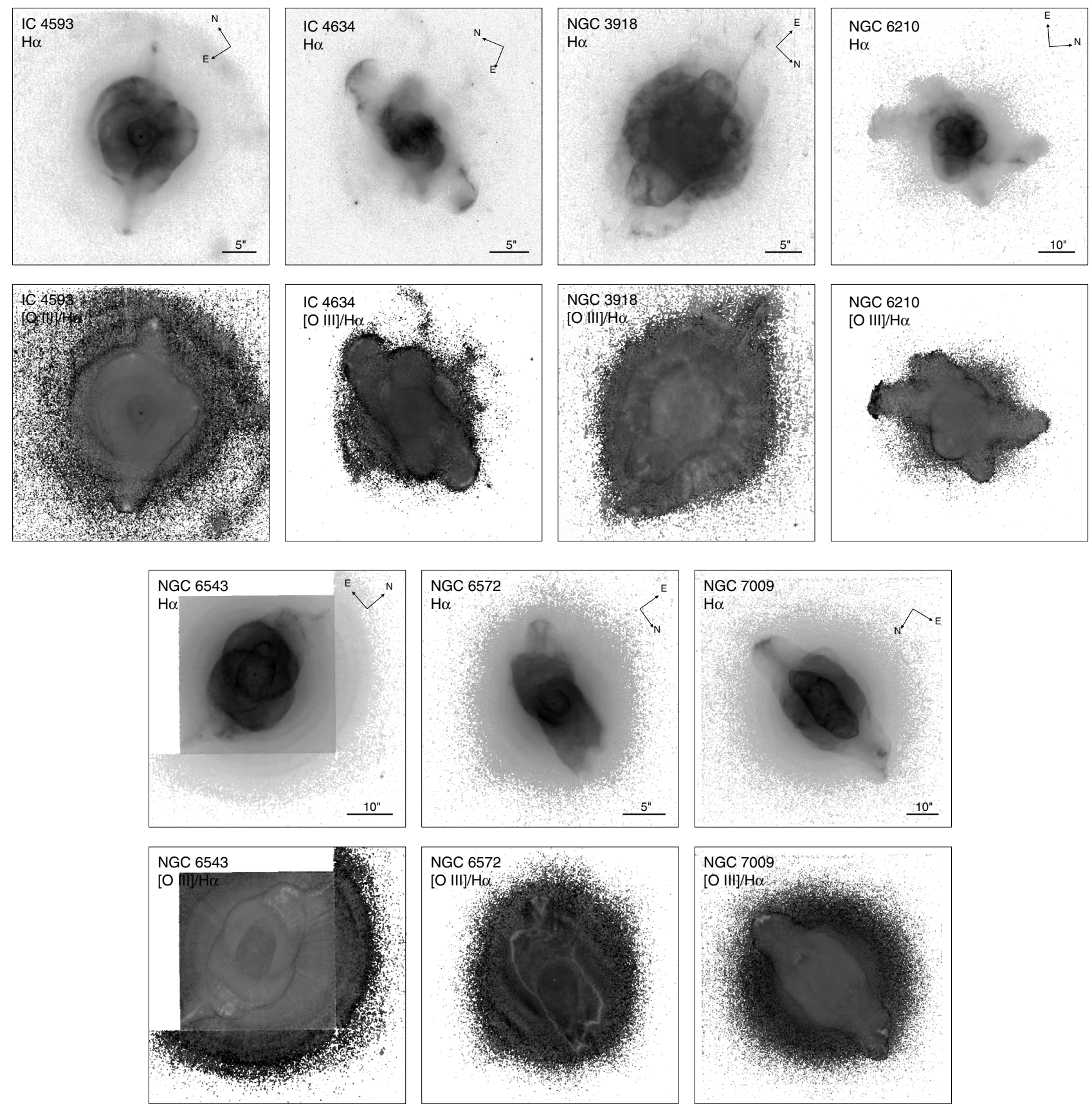

Fig. 1. $\mathrm{H} \alpha$ and $[\mathrm{O}$ III]/H $\alpha$ ratio maps of the type A PNe IC 4593, IC 4634, NGC 3918, NGC 6210, NGC 6543, NGC 6572, and NGC 7009. In the $[\mathrm{O} \mathrm{III}] / \mathrm{H} \alpha$ ratio maps, regions of relatively bright $\mathrm{H} \alpha$ emission appear bright (white), whereas regions of strong [O III] emission relative to $\mathrm{H} \alpha$ are shown dark (black).

and NGC 7662 whose $\mathrm{H} \alpha$ images and [O III]/H $\alpha$ ratio maps are shown in Fig. 2. Although some of these PNe have Fast Low-Ionization Emission Regions (the so-called FLIERs), the skins of enhanced $[\mathrm{O} \mathrm{III}] / \mathrm{H} \alpha$ ratios described here are not related to these features.

\section{- Type C}

$\mathrm{PNe}$ where the $[\mathrm{O} \mathrm{III}] / \mathrm{H} \alpha$ ratio decreases at the outer edge of the nebula, i.e., these $\mathrm{PNe}$ show the opposite behavior to type $\mathrm{B}$ PNe. Among these PNe are: IC 418, NGC 6720, BD+30³639, MyCn 18, Hen 3-1357, NGC 6881, NGC 6886, M 1-20, M2-14, M 2-43, and Hen 2-260. The $\mathrm{H} \alpha$ image and $[\mathrm{O}$ III] $/ \mathrm{H} \alpha$ ratio map of these PNe are shown in Fig. 3.
- Type D

PNe whose $[\mathrm{O} \mathrm{III}] / \mathrm{H} \alpha$ ratio maps do not show the effects described above (Fig. 4). Some of them have flat [O III]/H $\alpha$ ratio maps (e.g., Wray 16-286), some have low signalto-noise $(\mathrm{S} / \mathrm{N})$ ratio maps (e.g., M3-14), and some of them show too complex structures (e.g., NGC 2392 and NGC 6369) to be clearly ascribed to type A, B, or C.

The four different types described above are not mutually exclusive. In particular, most type A PNe are also type B, i.e., they show regions of enhanced $[\mathrm{O} \mathrm{III}] / \mathrm{H} \alpha$ ratios that are associated both with expanding nebular shells and bow-shock features produced by fast collimated outflows. For these PNe, we 

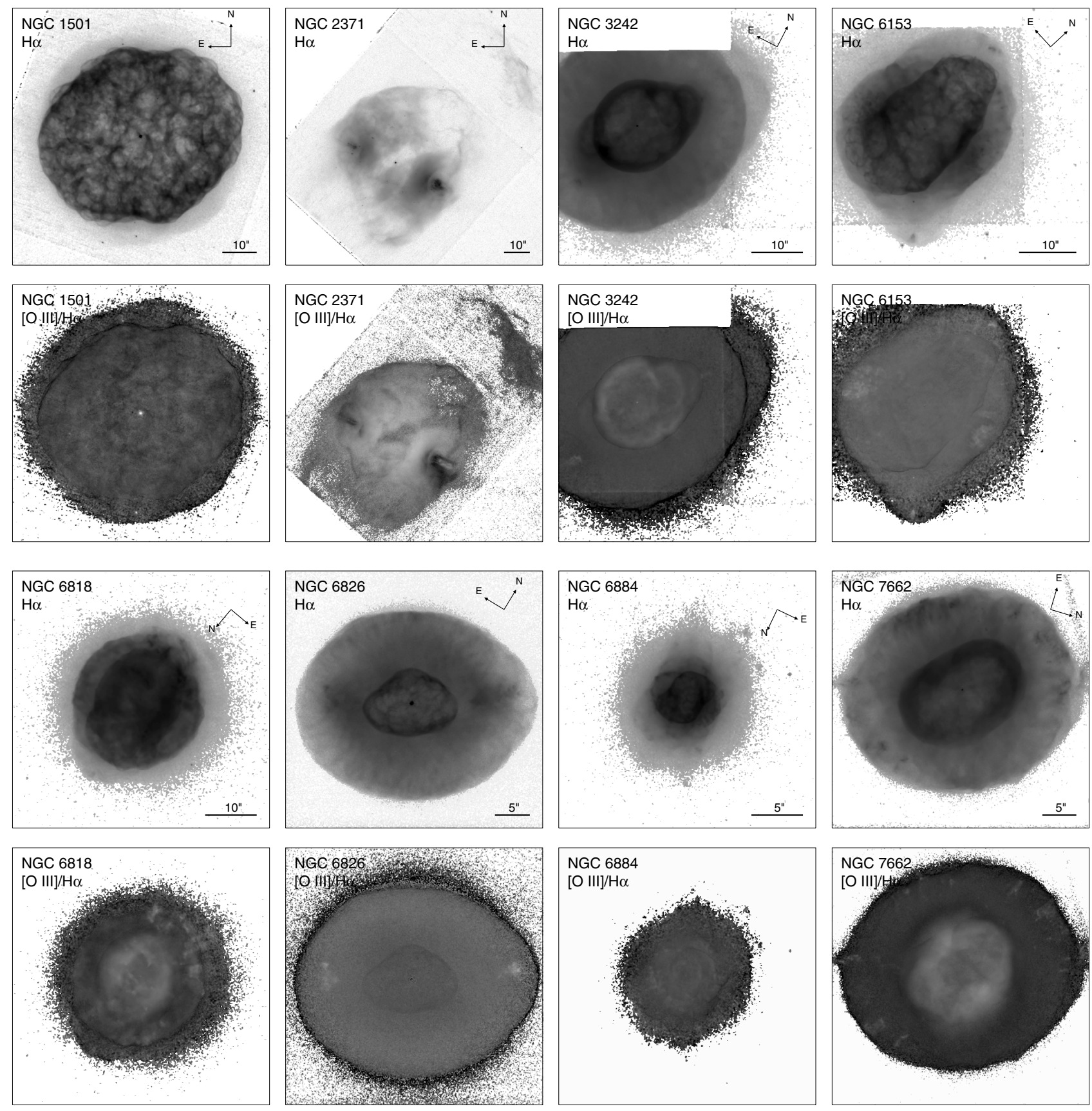

Fig. 2. Same as Fig. 1 for the type B PNe NGC 1501, NGC 2371-2, NGC 3242, NGC 6153, NGC 6818, NGC 6826, NGC 6884, and NGC 7662.

use the type AB. The only exception is that of NGC 6572, where a collimated outflow towards the north of the nebula produces a bow-shock feature with enhanced values of the $[\mathrm{O}$ III $] / \mathrm{H} \alpha$ ratio, whereas this ratio is diminished around the main nebular shell. For this PN, we use the type AC.

The $\mathrm{PNe}$ with adequate $[\mathrm{O} \mathrm{III}] / \mathrm{H} \alpha$ ratio maps are listed in Table 2. In this table we provide the classification of each PN and relevant information for a comparative study: the morphology or nebular shape $(\mathrm{E}=$ elliptical, $\mathrm{B}=$ bipolar, $\mathrm{MP}=$ quadrupolar or multi-polar, $\mathrm{PS}=$ point-symmetric, $\mathrm{MS}=$ multiple shell), the temperature of the central star, the nebular electron density, an averaged angular radius, the distance and linear radius, the [O III] $\lambda 5007$ and [N II] $\lambda 6584$ to $\mathrm{H} \beta$ line intensity ratios, and comments on the presence of collimated outflows, FLIERs, and ansae, or on the quality of the observations. Most of the data in this table have been obtained from Frew (2008). Additional references are given in the last column of Table 2.

\section{Discussion}

\subsection{Statistical properties}

A preliminary inspection of Table 2 provides hints of the varying nature of PNe belonging to the different types described in the previous section. These are further illustrated by the distributions of different parameters shown in Fig. 5.

Type D PNe is an heterogeneous group severely affected by several observational biases. Most type D PNe are small, with angular radii $\leq 4^{\prime \prime}$, and linear sizes $\leq 0.03 \mathrm{pc}$. The small size of these objects is clearly introducing an observational bias in 
Martín A. Guerrero et al.: Unveiling shocks in PNe
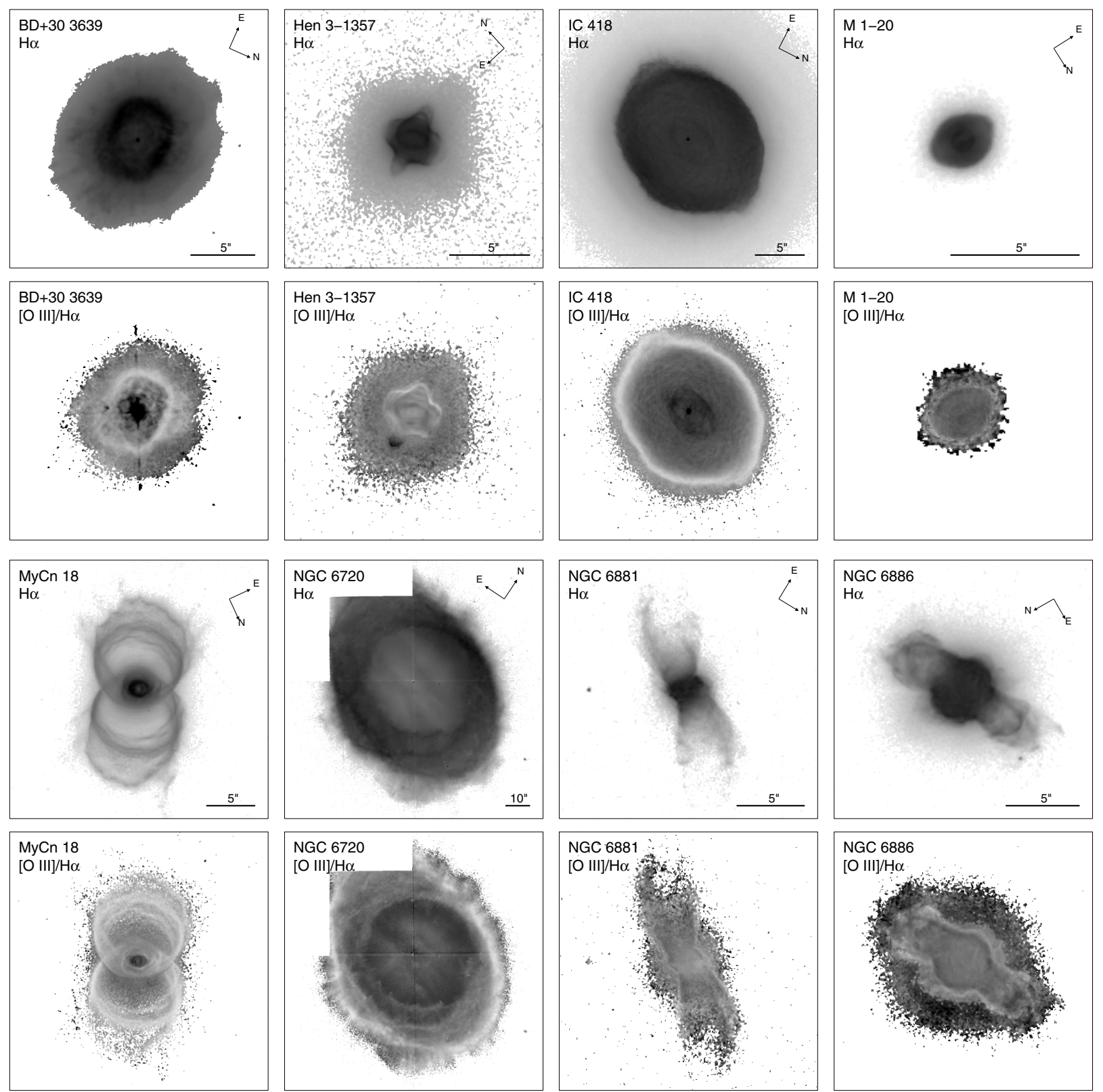

Fig. 3. Same as Fig. 1 for the type C PNe.

the sample, as the fine morphological details of these objects cannot be properly resolved. Furthermore, the $\mathrm{S} / \mathrm{N}$ of the images of a significant number of type D PNe is limited, thus hampering the detection of structures with contrasting values of the $[\mathrm{O} \mathrm{III}] / \mathrm{H} \alpha$ ratio. On the other hand, some objects in this group are large and well resolved, but they have very complex morphologies (e.g., NGC 2392 and NGC 6369). Although these sources have low [N II] $\lambda 6584 \AA$ to $\mathrm{H} \alpha$ integrated line ratios, the presence of low-excitation features may result in local regions of significant [N II] emission. The [N II] contamination on the $\mathrm{H} \alpha$ would correspond to features of apparently diminished values of the $[\mathrm{O} \mathrm{III}] / \mathrm{H} \alpha$ ratio. The complexity of the ratio maps may hide the possible effects of the expansion of nebular shells and collimated outflows in the surrounding material.

$\mathrm{PNe}$ of type $\mathrm{C}$ also tend to be small, with radii and linear sizes similar to, but slightly larger than, those of type D PNe. Notably, some of the type C PNe (e.g., NGC2346 and

NGC 6720) have the largest angular radii and linear sizes among the PNe in this sample. In contrast to type D PNe, the distribution of the effective temperature of the central stars of type C PNe clearly peaks at low values $\left(T_{\text {eff }} \leq 50000 \mathrm{~K}\right)$. PNe of type C are either young, with low $T_{\text {eff }}$ central stars (e.g., BD+30 3639 and IC 418), or have hot central stars which have already turned towards the white dwarf cooling track and reduced their stellar luminosities (e.g., the evolved PN NGC 6720 and the prominent bipolar PNe NGC 2346 and NGC 6886, Napiwotzki 1999; Méndez \& Niemela 1981; Pottasch \& Surendiranath 2005). In the first case, the stellar spectrum is not hard enough to maintain the oxygen twice ionized throughout the whole nebula, whereas in the second case the spectrum is very hard but the photon flux seen by material at large distances from the central star is small. In both cases, the ionization degree drops at the edge of the nebula and an exterior $\mathrm{O}^{+}$region forms. Indeed, the reduced number of type C PNe with available HST WFPC2 images in the 

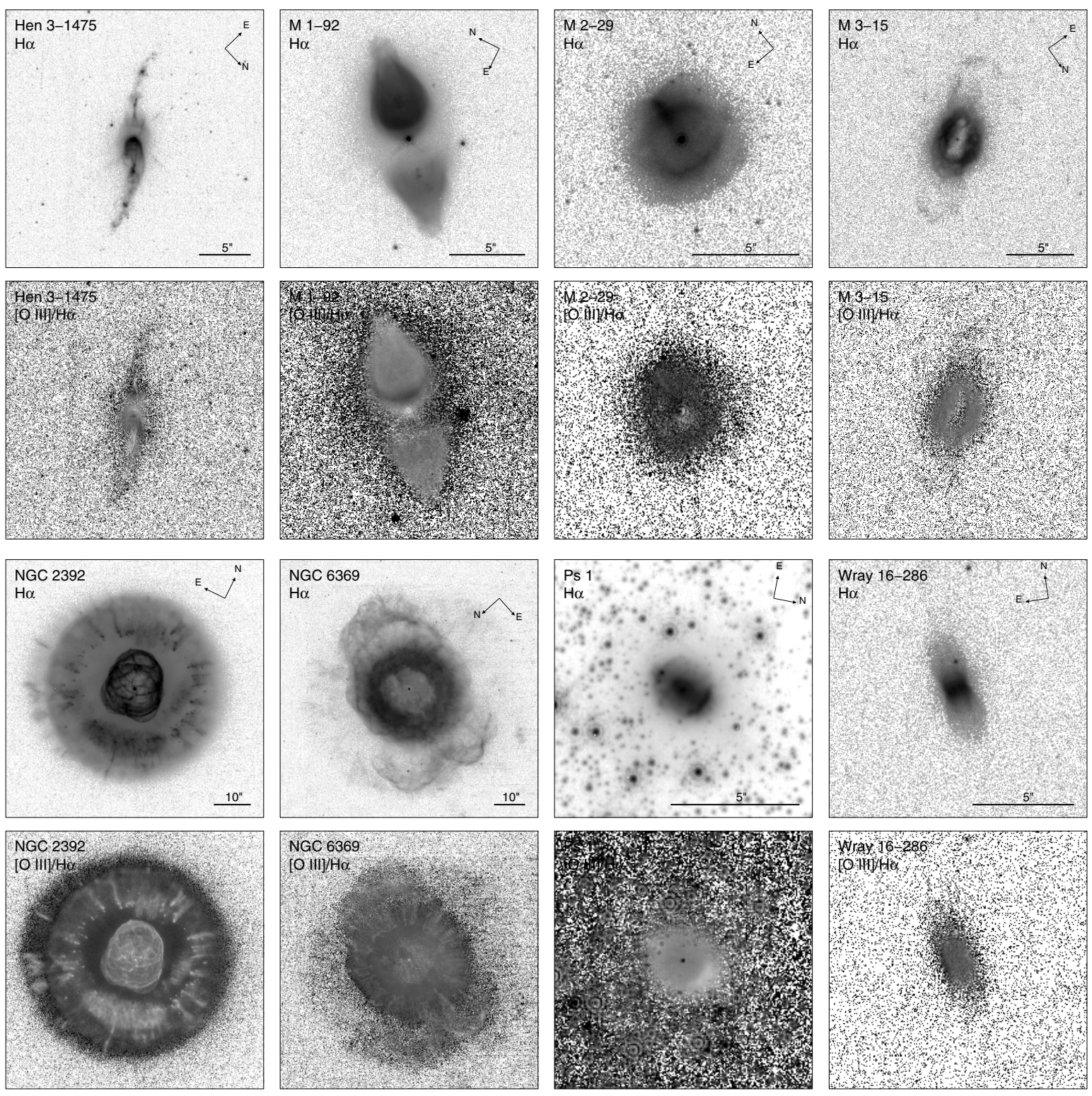

Fig. 4. Same as Fig. 1 for a subsample of type D PNe.

[O II] $\lambda 3727 \AA$ A line consistently reveal bright emission in regions of low $[\mathrm{O} \mathrm{III}] / \mathrm{H} \alpha$. The corresponding high value of the $[\mathrm{O} \mathrm{II}] /[\mathrm{O}$ III] line ratio in these regions can be interpreted as a sudden drop in the ionization degree. The decrease in the ionization degree will also enhance the emission of the low-excitation $[\mathrm{N}$ II] lines. Therefore, the reduced $[\mathrm{O}$ III] $/ \mathrm{H} \alpha$ values observed in the ratio maps of IC 418 (Ramos-Larios et al. 2012) and NGC 6720 (O'Dell et al. 2013) among others can be partially ascribed to the local contaminant contribution in these regions of [N II] emission on the $\mathrm{H} \alpha$ image.

PNe of type A and B are, on average, better resolved than type $\mathrm{C} P \mathrm{Pe}$, with angular radii spanning between $\sim 4 "$ and $\sim 20^{\prime \prime}$, and linear sizes greater than $0.02 \mathrm{pc}$, peaking at $\sim 0.1 \mathrm{pc}$. The distribution of the effective temperature of their central stars (median value $\sim 75000 \mathrm{~K}$ ) is somehow similar to that of type $\mathrm{C} P \mathrm{Pe}$ (median value $\sim 60000 \mathrm{~K}$ ). More obvious differences are revealed by the distributions of their $[\mathrm{N} \mathrm{II}] /[\mathrm{O}$ III] ratios: those of type A and B peak at low values of the $[\mathrm{N} \mathrm{III}] /[\mathrm{O}$ III] ratio, with most data points below 0.1 , whereas the distribution of type $\mathrm{C}$ PNe shows a notable tail towards high values of the $[\mathrm{N}$ II $] /[\mathrm{O}$ III] ratio. If we could exclude abundances effects, this

result would imply that type $\mathrm{C}$ PNe have lower excitation than type A and B PNe. Finally, we remark that the occurrence of multiple-shell morphology and collimated outflows or FLIERs (suggestive of fast collimated outflows, although with expansion velocities comparable to these of the nebular shells) is the highest among PNe of type A and B.

\section{2. $[\mathrm{O} I I 1] / \mathrm{H} \alpha$ radial profiles}

To investigate in more detail the physical properties of the regions where the variations of the $[\mathrm{O} \mathrm{III}] / \mathrm{H} \alpha$ ratio take place, we have extracted radial profiles of representative PNe of types A, B, and C. These radial profiles, shown in Figs. 6-8, respectively, have been derived from flux calibrated HST images; however, we have not attempted to deredden the line ratios and, therefore, the values of the $[\mathrm{O} \mathrm{III]}] / \mathrm{H} \alpha$ ratio in these profiles do not correspond to absolute line intensity ratios. The relative enhancement (or decrement for type C PNe) of the $[\mathrm{O} \mathrm{III]}] / \mathrm{H} \alpha$ ratio and the width of the region with anomalous [O III] emission derived from these profiles are listed in Table 3. 


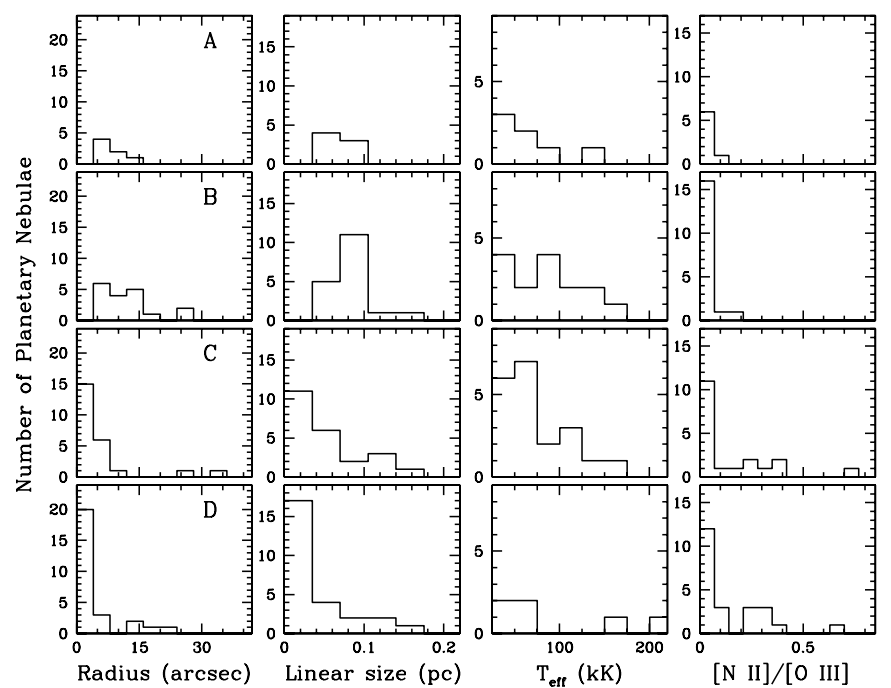

Fig. 5. Distributions of the angular radii, linear sizes, $[\mathrm{N}$ II $] /[\mathrm{O}$ III $]$ ratios, and stellar effective temperatures of the different types of $\mathrm{PNe}$ defined in Sect. 3.

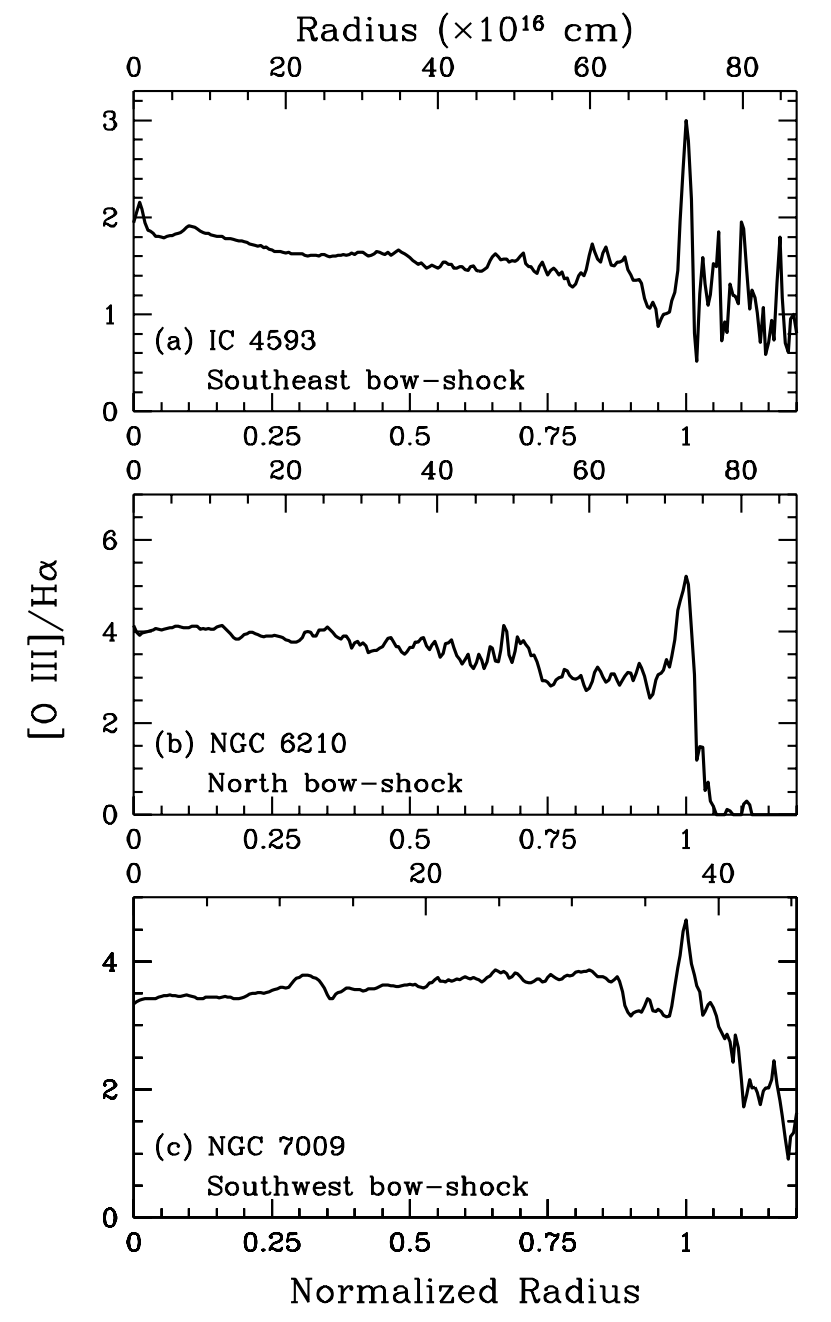

Fig. 6. Radial profiles of the $[\mathrm{O} \mathrm{III}] / \mathrm{H} \alpha$ ratio maps of the type A PNe IC 4593, NGC 6210, and NGC 7009 along selected bow-shock features associated with collimated outflows.

The regions of enhanced $[\mathrm{O} \mathrm{III}] / \mathrm{H} \alpha$ ratio associated with bow-shocks, outer shells, and blisters show the largest increase

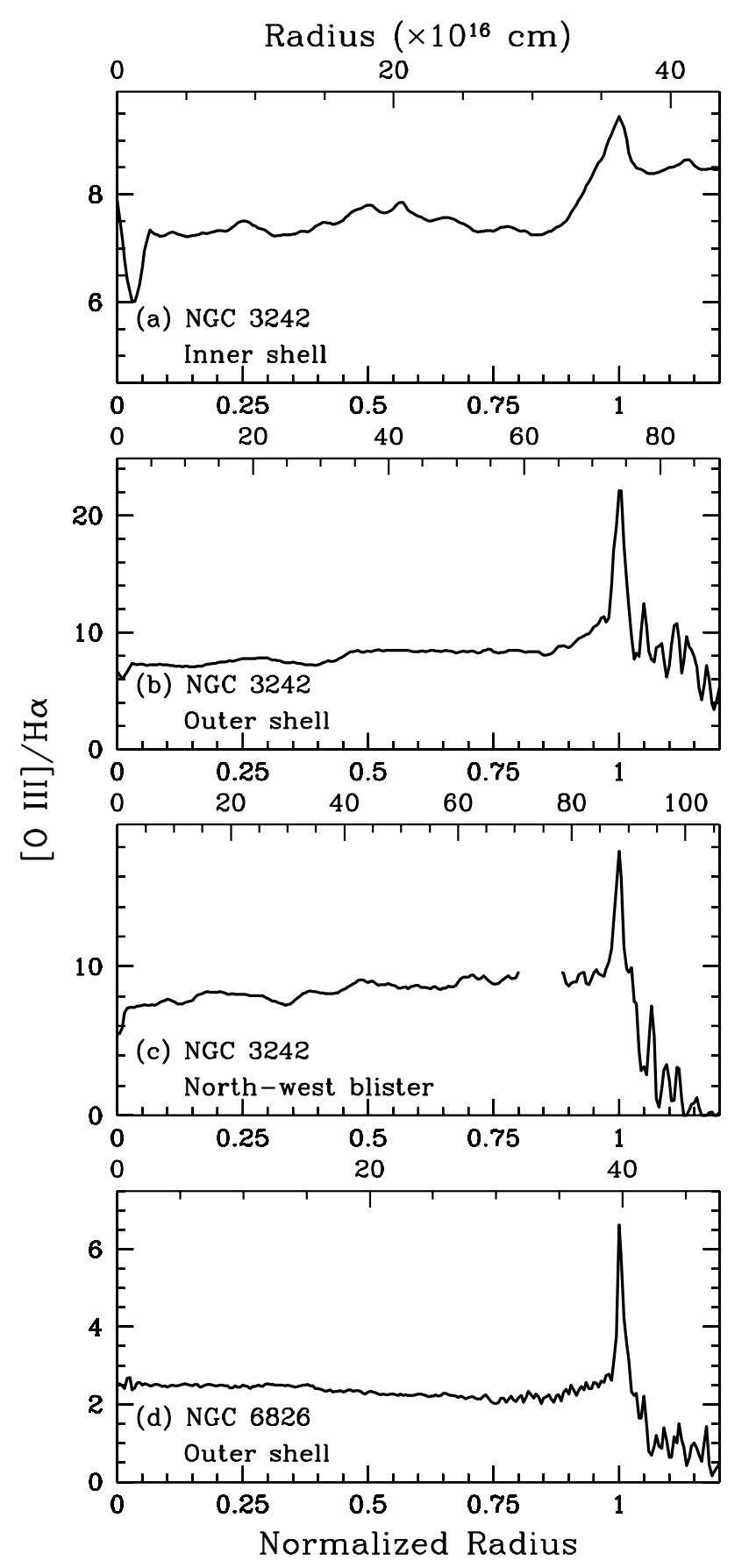

Fig. 7. Radial profiles of the $[\mathrm{O} \mathrm{III}] / \mathrm{H} \alpha$ ratio maps of the type $\mathrm{B} \mathrm{PNe}$ NGC 3242 a), b), c) and NGC 6826 d). The radial profiles of NGC 3242 correspond to the inner shell $\mathbf{a}$ ), the outer shell b), and the north-west blister of the outer shell c). The radial profile of NGC 6826 d) corresponds to the outer shell. The $[\mathrm{O} \mathrm{III}] / \mathrm{H} \alpha$ peaks of the outer shell of NGC 3242 are not shown in the profile of the blister of this shell c).

of this ratio, being typically larger by a factor of two. On the contrary, the enhancement of the $[\mathrm{O} \mathrm{III}] / \mathrm{H} \alpha$ ratio at the inner shells of type B PNe is typically smaller. Those inner shells are embedded within the corresponding outer shells, and thus we interpret that the smaller enhancement of the $[\mathrm{O} \mathrm{III}] / \mathrm{H} \alpha$ ratio is the result of the dilution of the emission from the skin of high $[\mathrm{O}$ III $] / \mathrm{H} \alpha$ ratio by the emission from the outer shell.

There are also notable differences among the fractional sizes of these regions. The peaks of enhanced [O III] emission in bowshocks, outer shells, and blisters show the narrowest fractional 


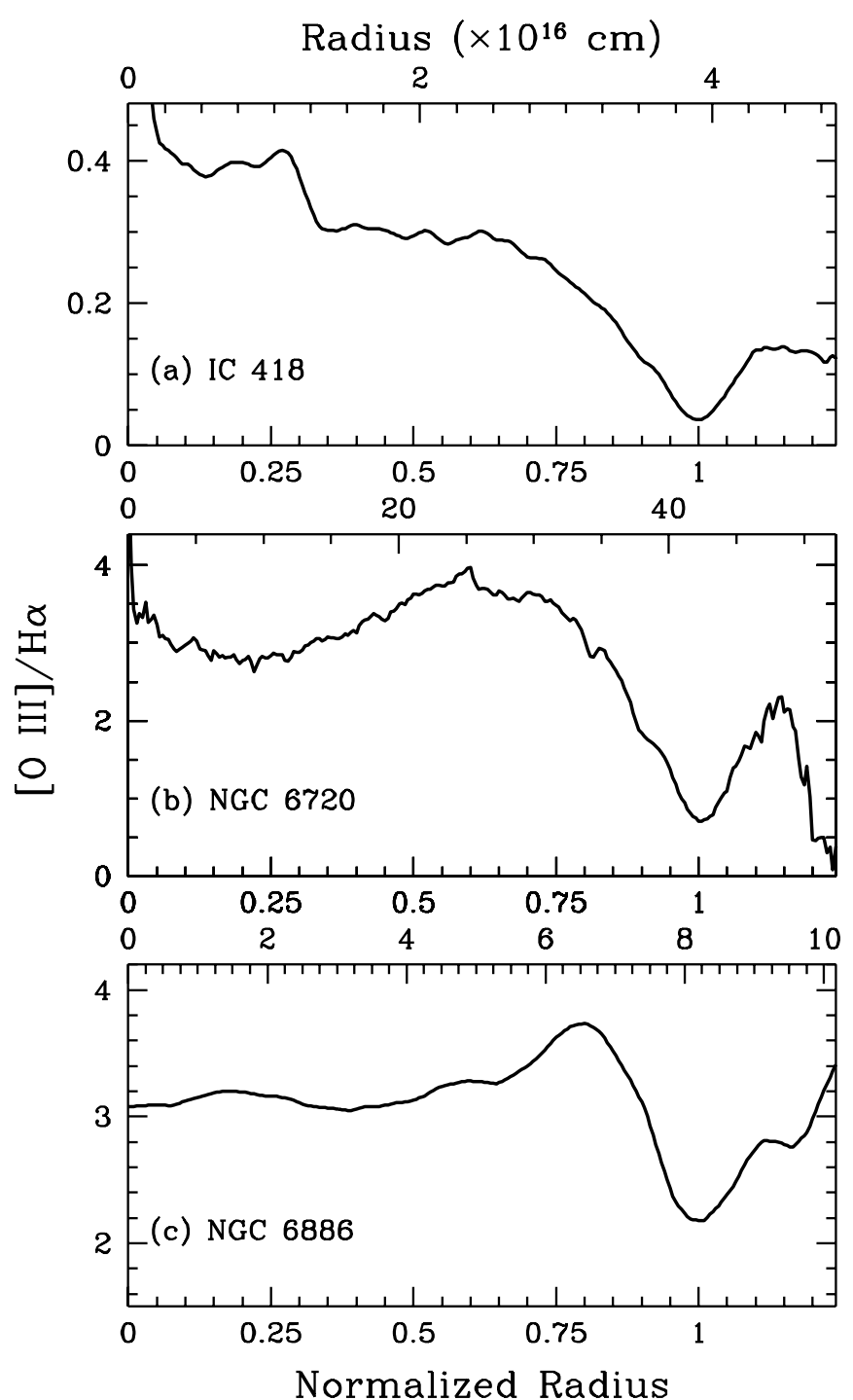

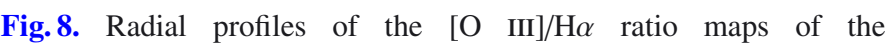
type C PNe IC 418, NGC 6720, and NGC 6886 along their minor axes at $\mathrm{PA} \sim 70^{\circ}, 130^{\circ}$, and $45^{\circ}$, respectively.

sizes, all being $\leq 4 \%$. Meanwhile, the regions of enhanced $[\mathrm{O}$ III] $] / \mathrm{H} \alpha$ ratios associated with the inner shell of NGC 3242 and the absorptions in the $[\mathrm{O} \mathrm{III}] / \mathrm{H} \alpha$ radial profiles of type $\mathrm{C}$ PNe are broader, with fractional widths above $10 \%$. The physical sizes of all these regions are rather similar, $\approx 10^{16} \mathrm{~cm}$, but for the outer shell of NGC 6826, which is especially thin $\left(4 \times 10^{15} \mathrm{~cm}\right)$. Even the outer regions of diminished [O III] $/ \mathrm{H} \alpha$ ratios of type C have similar physical sizes, except for NGC 6720, which is especially broad $\left(\approx 10^{17} \mathrm{~cm}\right)$.

There are two interesting features in these radial profiles which are worth mentioning as they are indicative of the variations in excitation affecting the nebular material and of the geometry of these regions. First, the radial profiles of bow-shocks in Fig. 6 display a depression just inside the emission peak which is then followed by a bounce in this ratio further in. The regions of diminished values in the $[\mathrm{O} \mathrm{III}] / \mathrm{H} \alpha$ ratio are associated with the prominent low-ionization features found inside these bow-shocks, whereas the increase in the value of the line ratio suggests a relative brightening of the [O III] emission on larger spatial scales than the outer skin. Secondly, the $[\mathrm{O}$ III $] / \mathrm{H} \alpha$ peak associated with outer shells is preceded by a ramp in this ratio, as can be seen in the radial profiles of the outer shells of NGC 3242 and NGC 6826 (Fig. 7). Projection effects can be responsible for this feature.

\subsection{Origin of the high $[\mathrm{O} I I I] / H \alpha$ skins}

As mentioned in Sect. 4.1, the $[\mathrm{O} \mathrm{III}] / \mathrm{H} \alpha$ ratio maps of type $\mathrm{C} \mathrm{PNe}$ can be explained in terms of a nebular ionization structure in which the $\mathrm{O}^{++} / \mathrm{H}^{+}$ratio decreases in the outermost nebular layers. The low effective temperature of the central stars of young PNe or the low luminosity of the central stars of bipolar or evolved $\mathrm{PNe}$ may result in these low ionization degrees.

Similarly, one might seek an explanation to the $[\mathrm{O}$ III] $] / \mathrm{H} \alpha$ ratio maps of type $\mathrm{A}$ and $\mathrm{B}$ PNe in terms of a nebular ionization structure: the outward increase in the [O III] emission would be caused by a decrease of the $\mathrm{O}^{3+} / \mathrm{H}^{+}$ratio and a subsequent increase in the $\mathrm{O}^{++} / \mathrm{H}^{+}$ratio. If this were the case, one would expect low [O III] emission throughout the nebula and its brightening at the outer edge, mimicking the structure of the [O II] emission of type $\mathrm{C}$ PNe. However, it turns out that the brightness of the [O III] $\lambda 5007 \AA$ line is consistently high throughout the whole nebula in both type A and B PNe. Furthermore, the $\mathrm{O}^{3+} / \mathrm{O}^{++}$fraction required to reproduce such a structure would require stellar temperature and luminosities much larger than those typical of $\mathrm{PNe}$ (the maximum $\mathrm{O}^{3+} / \mathrm{O}^{++}$ values observed in $\mathrm{PNe}$ are about 0.5 ). This indicates that the local enhancement of the $[\mathrm{O} \mathrm{III}] / \mathrm{H} \alpha$ ratio in type $\mathrm{A}$ and $\mathrm{B} \mathrm{PNe}$ is most probably caused by a sharp increase in temperature, which cannot be reproduced by photoionization models, unless an ad hoc additional heating mechanism working on small spatial scales produces a sharp raise in $T_{\mathrm{e}}$. Such an increase in $T_{\mathrm{e}}$ would reduce the effective recombination rate of $\mathrm{H} \alpha$, which is roughly proportional to $T_{\mathrm{e}}^{-0.9}$, and increase the collisional excitation of $\mathrm{O}^{++}$, which is roughly proportional to $T^{-1 / 2} \mathrm{e}^{-E / k T}$. The $[\mathrm{O} \mathrm{III}] / \mathrm{H} \alpha$ line intensity ratio can be expressed as:

$I_{[\mathrm{O} \text { III] }]} / I_{\mathrm{H} \alpha} \propto \frac{N\left(\mathrm{O}^{++}\right)}{N\left(\mathrm{H}^{+}\right)} T_{\mathrm{e}}^{0.4} \mathrm{e}^{-28000 / T_{\mathrm{e}}}$.

We plot in Fig. 9 the variation of the $[\mathrm{O}$ III $] / \mathrm{H} \alpha$ line intensity ratio with the increase of electron temperature above a baseline temperature in the range typical of PNe, $8000 \mathrm{~K} \leq T_{\mathrm{e}} \leq$ $12000 \mathrm{~K}$. We note that these curves have not been computed using the above expression, but the exact line emissivities. For a baseline temperature of the nebular material of $10000 \mathrm{~K}$, the observed increase of the $[\mathrm{O} \mathrm{III}] / \mathrm{H} \alpha$ line intensity ratio by factors of 2 to 3 implies a jump in $T_{\mathrm{e}}$ of $2000-5000 \mathrm{~K}$.

The extra heating can be provided by a forward shock propagating into a tenuous medium (Cox 1972) as it has been observed in collimated outflows in Herbig-Haro objects (e.g., HH 34, Rodríguez-González et al. 2012) and ring nebulae around WR stars (Gruendl et al. 2000). The extra heating in type A PNe is provided by the forward shock of a fast outflow propagating into the tenuous, outer regions of the $\mathrm{PNe}$ or the interstellar medium (ISM). The shocks produced by collimated outflows in Herbig-Haro objects enhance the emission of low-ionization lines (e.g., [O I], [S II], or [N II]), as predicted for steady, plane-parallel shock models (Hartigan et al. 1987), but the situation is notably different in PNe. Their outflows are photoionized by the central star, and this results in variations of the expected line ratios and spatial distribution of the emission from different lines that has been modeled for fast, compact knots traveling away from the central star by Raga et al. (2008). In particular, these models predict a relative brightening of the [O III] emission with respect to that of $\mathrm{H} \alpha$ at larger distances 
Table 3. Quantitative properties of the $[\mathrm{O}$ III] $] / \mathrm{H} \alpha$ profiles of Figs. 6-8.

\begin{tabular}{|c|c|c|c|c|c|}
\hline \multirow[t]{2}{*}{ PN } & \multirow[t]{2}{*}{ Region } & \multirow{2}{*}{$\begin{array}{c}{[\mathrm{O} \mathrm{III}] / \mathrm{H} \alpha} \\
\text { enhancement }\end{array}$} & \multicolumn{3}{|c|}{$F W H M^{a}$} \\
\hline & & & $(\%)$ & $\left({ }^{\prime \prime}\right)$ & $(\mathrm{cm})$ \\
\hline \multicolumn{6}{|c|}{ Type A PNe } \\
\hline IC 4593 & Southern bow-shock & $\times 2.0$ & $2 \%$ & 0.27 & $1.5 \times 10^{16}$ \\
\hline NGC 6210 & Northern bow-shock & $\times 1.7$ & $3 \%$ & 0.70 & $2.1 \times 10^{16}$ \\
\hline NGC 7009 & Eastern bow-shock & $\times 1.3$ & $3 \%$ & 0.63 & $1.1 \times 10^{16}$ \\
\hline \multicolumn{6}{|c|}{ Type B PNe } \\
\hline \multirow[t]{3}{*}{ NGC 3242} & Inner shell & $\times 1.3$ & $10 \%$ & $0 ! 93$ & $3.6 \times 10^{16}$ \\
\hline & Outer shell & $\times 2.7$ & $3 \%$ & 0.57 & $2.2 \times 10^{16}$ \\
\hline & North-west blister & $\times 2.0$ & $1.5 \%$ & 0.38 & $1.5 \times 10^{16}$ \\
\hline NGC 6826 & Outer shell & $\times 3.1$ & $1 \%$ & 0.14 & $4.0 \times 10^{15}$ \\
\hline \multicolumn{6}{|c|}{ Type C PNe } \\
\hline IC 418 & Main shell & $\div 6.0$ & $40 \%$ & 1."7 & $1.5 \times 10^{16}$ \\
\hline NGC 6720 & Main shell & $\div 4.9$ & $24 \%$ & $7 . .5$ & $1.0 \times 10^{17}$ \\
\hline NGC 6886 & Main shell & $\div 1.7$ & $18 \%$ & 0.32 & $1.5 \times 10^{16}$ \\
\hline
\end{tabular}

Notes. ${ }^{(a)}$ The shape of the profile of these regions is not symmetric and the measurement of their widths corresponds to the innermost half of the profile.

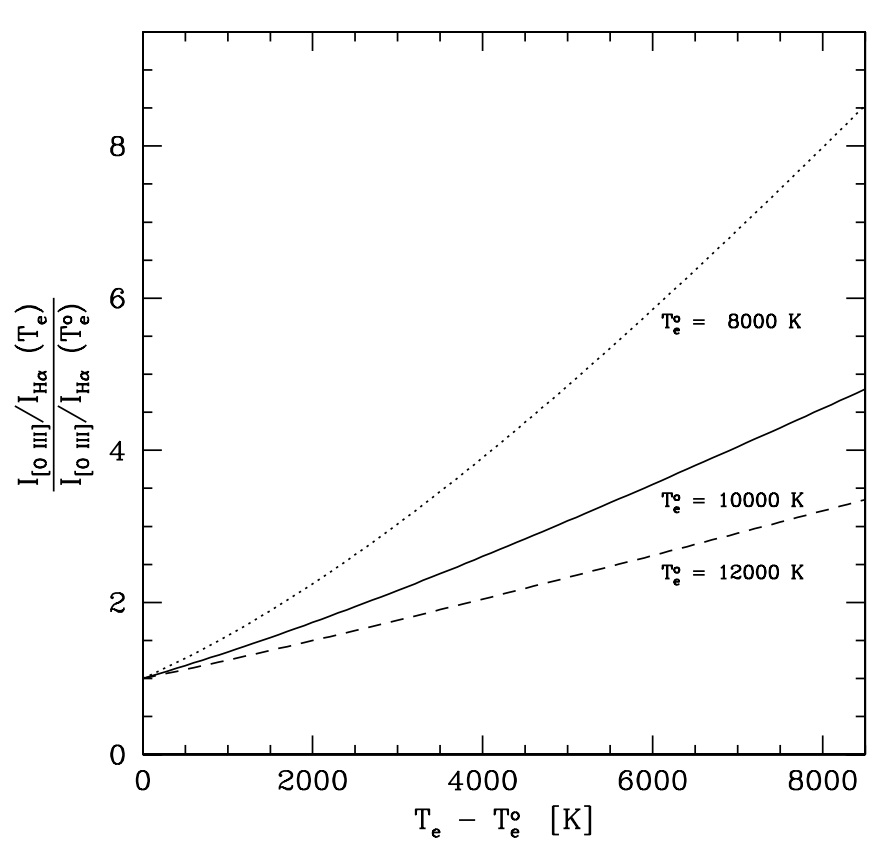

Fig. 9. Variation of the $[\mathrm{O} \mathrm{III}] / \mathrm{H} \alpha$ line intensity ratio associated with the increase in electron temperature from $T_{\mathrm{e}}^{\mathrm{o}}$ to $T_{\mathrm{e}}$. Three different curves are plotted, depending on the value of the pre-shock temperature.

from the ionizing source (their Fig. 9), in clear agreement with the radial profiles displayed in Fig. 6.

Meanwhile, the expansion of a higher density shell into a lower density, outer shell or into the ISM produces a forward shock in type B PNe which heats the material in front of the shell. In this case, the pre-shock material is already highly ionized by the central star of the PN, and no enhancement of the emission from low-ionization lines is expected. Therefore, the analysis of $[\mathrm{O} \mathrm{III}] / \mathrm{H} \alpha$ ratio maps provides the only strategy to investigate the occurrence of the shocks associated to expanding shells of PNe.

Hydrodynamical models of PN evolution indeed predict the development of two different shocks during their formation (e.g., Perinotto et al. 2004; Schönberner et al. 2005). The sudden ionization of the material ejected during the AGB produces a D-type ionization front at the leading edge of the outer nebular shell of multiple-shell PNe. This shock propagates outwards, into the ambient AGB wind, and a thin skin of shocked material is expected at the leading shock of the shell. Model simulations of the propagation of such shock kindly performed by Schönberner and Steffen suggest that the post-shock temperatures depend very sensitively on the density jump across the shock, although a high numerical resolution is required to resolve the shock. Similarly, another shock is set by the wind interaction at the discontinuity between the outer nebular shell and the bright rim pushed by the thermal pressure of the hot bubble. The available model simulations predict the formation of a thick rim early in the evolution of the PN, resulting in a shock too weak to produce the observed enhancement of the $[\mathrm{O} \mathrm{III}] / \mathrm{H} \alpha$ ratios. With time, the rim sharpens and moves faster, and its leading shock becomes stronger (Perinotto et al. 2004).

These detailed hydrodynamical simulations determine that the velocity jump across the shock front at the leading edge of the outer shell is $5-40 \mathrm{~km} \mathrm{~s}^{-1}$. Across the shock front between the inner rim and the shell, the velocity jump is smaller, $\leq 10 \mathrm{~km} \mathrm{~s}^{-1}$. For typical electron densities of $\sim 4000 \mathrm{~cm}^{-3}, \sim 1000 \mathrm{~cm}^{-3}$, and $\sim 50 \mathrm{~cm}^{-3}$ for the rim, outer shell, and surrounding unperturbed AGB wind, these velocity jumps imply temperature increases $\simeq 4000 \mathrm{~K}$ which can easily produce the observed enhancement of the $[\mathrm{O}$ III] $/ \mathrm{H} \alpha$ ratios.

\section{Summary}

Using archival HST [O III] and $\mathrm{H} \alpha$ images of PNe, we have found regions of enhanced values of the $[\mathrm{O} \mathrm{III}] / \mathrm{H} \alpha$ ratio. These regions can be described as thin skins which envelop either bowshock features associated with fast collimated outflows PNe or nebular shells of PNe with multiple-shell morphology. The enhancement of the $[\mathrm{O} \mathrm{III}] / \mathrm{H} \alpha$ ratio is interpreted to be caused by a local increase of $T_{\mathrm{e}}$ which brightens the emission in the [O III] line and damps the emissivity of the $\mathrm{H} \alpha$ line. The amplitude of the variations in the values of the $[\mathrm{O}$ III] $/ \mathrm{H} \alpha$ ratio implies variations in $T_{\mathrm{e}}$ of $2000-5000 \mathrm{~K}$. The increase in $T_{\mathrm{e}}$ is associated with forward shocks produced by the expansion of collimated outflows and nebular shells into surrounding media of lower density. These results illustrate the complexity of PNe and its suitability to studying the detailed physics of ionized plasmas. 
Acknowledgements. All of the data presented in this paper were obtained from the Mikulski Archive for Space Telescopes (MAST). STScI is operated by the Association of Universities for Research in Astronomy, Inc., under NASA contract NAS5-26555. Support for MAST for non-HST data is provided by the NASA Office of Space Science via grant NNX09AF08G and by other grants and contracts. This work is funded by grants AYA 2005-01495 of the Spanish MEC (Ministerio de Educación y Ciencia), grant FGM-1747 of the Junta de Andalucía, and CSIC grant 2006-50-I135. M.A.G. and J.A.T. are supported by the Spanish MICINN (Ministerio de Ciencia e Innovación) grant AYA 201129754-C03-02, V.L. by grant AYA 2011-22614, L.F.M. by grant AYA 201130228-C03-01, and A.R. by grant AYA 2011-30228-C03, all of them co-funded with FEDER funds. J.A.T. acknowledges support by the CSIC JAE-Pre student grant 2011-00189, and PVF by CONACyT grant 167611 and DGAPA-PAPIIT (UNAM) grant IN105312. We kindly thanks Detlef Schönberner and Matthias Steffen for helpful discussion.

\section{References}

Acker, A., Raytchev, B., Koeppen, J., \& Stenholm, B. 1991, A\&AS, 89, 237

Acker, A., Marcout, J., Ochsenbein, F., et al. 1992, The Strasbourg-ESO Catalogue of Galactic Planetary Nebulae. Parts I, II., eds. A. Acker, J. Marcout, F. Ochsenbein, et al., European Southern Observatory, Garching (Germany), 1047

Bässgen, M., Diesch, C., \& Grewing, M. 1990, A\&A, 237, 201

Balick, B. 2004, AJ, 127, 2262

Balick, B., \& Frank, A. 2002, ARA\&A, 40, 439

Bohigas, J. 2001, Rev. Mex. Astron. Astrophys., 37, 237

Cavichia, O., Costa, R. D. D., \& Maciel, W. J. 2010, Rev. Mex. Astron. Astrophys., 46, 159

Corradi, R. L. M., Manso, R., Mampaso, A., \& Schwarz, H. E. 1996, A\&A, 313, 913

Corradi, R. L. M., Guerrero, M., Manchado, A., \& Mampaso, A. 1997a, New Astron., 2, 461

Corradi, R. L. M., Perinotto, M., Schwarz, H. E., \& Claeskens, J.-F. 1997b, A\&A, 322, 975

Corradi, R. L. M., Sabin, L., Miszalski, B., et al. 2011, MNRAS, 410, 1349

Cox, D. P. 1972, ApJ, 178, 143

Dennis, T. J., Cunningham, A. J., Frank, A., et al. 2008, ApJ, 679, 1327

Ercolano, B., Morisset, C., Barlow, M. J., Storey, P. J., \& Liu, X.-W. 2003 , MNRAS, 340, 1153

Ercolano, B., Wesson, R., Zhang, Y., et al. 2004, MNRAS, 354, 558

Frew, D. 2008, Ph.D. Thesis, Macquarie University, Sydney, Australia

Gruendl, R. A., Chu, Y.-H., Dunne, B. C., \& Points, S. D. 2000, AJ, 120, 2670

Guerrero, M. A., Miranda, Luis F., Riera, Angels, et al. 2008, ApJ, 683, 272

Guiles, S., Bernard-Salas, J., Pottasch, S. R., \& Roellig, T. L. 2007, ApJ, 660, 1282

Hartigan, P., Raymond, J., \& Hartmann, L. 1987, ApJ, 316, 323

Hyung, S., \& Aller, L. H. 1996, MNRAS, 278, 551

Hyung, S., \& Aller, L. H. 1997, ApJ, 491, 242
Hyung, S., Aller, L. H., \& Feibelman, W. A. 1994a, MNRAS, 269, 975 Hyung, S., Aller, L. H., \& Feibelman, W. A. 1994b, PASP, 106, 745

Hyung, S., Aller, L. H., \& Feibelman, W. A. 1997, ApJS, 108, 503

Hyung, S., Aller, L. H., \& Feibelman, W. A. 1999, ApJ, 525, 294

Hyung, S., Aller, L. H., Feibelman, W. A., \& Lee, S.-J. 2001, ApJ, 563, 889

Kwitter, K. B., \& Henry, R. B. C. 1998, ApJ, 493, 247

Lee, C. F., \& Sahai, R. 2003, ApJ, 586, 319.

Lim, P. L., Quijada, M., Baggett, S., et al. 2010, Space Telescope Science Institute, Instrument Science Report WFPC2 2010-05

Méndez, R. H., \& Niemela, V. S. 1981, ApJ, 250, 240

Méndez, R. H., Kudritzki, R. P., Herrero, A., Husfeld, D., \& Groth, H. G. 1988, A\&A, 190, 113

Moore, B. D., Walter, D. K., Hester, J. J., et al. 2002, AJ, 124, 3313

Napiwotzki, R. 1999, A\&A, 350, 101

O'Dell, C. R., Ferland, G. J., Henney, W. J., \& Peimbert, M. 2013, AJ, 145, 92

Perinotto, M., Schönberner, D., Steffen, M., \& Calonaci, C. 2004, A\&A, 414, 993

Phillips, J. P. 2004, MNRAS, 353, 589

Phillips, J. P., \& Cuesta, L. 1998, A\&AS, 133, 381

Pottasch, S. R., \& Surendiranath, R. 2005, A\&A, 432, 139

Pottasch, S. R., Gathier, R., Gilra, D. P., \& Wesselius, P. R. 1981, A\&A, 102, 237

Pottasch, S. R., Bernard-Salas, J., Beintema, D. A., \& Feibelman, W. A. 2004, A\&A, 423, 593

Pottasch, S. R., Beintema, D. A. \& Feibelman, W. A. 2005, A\&A, 436, 953

Pottasch, S. R., Bernard-Salas, J., \& Roellig, T. L. 2009, A\&A, 499, 249

Raga, A. C., Velázquez, P. F., Cantó, J., \& Masciadri, E. 2002, A\&A, 395, 647

Raga, A.C., Riera, A., Mellema, G., Esquivel, A., \& Velázquez, P. F. 2008, A\&A, 489,1141

Ramos-Larios, G., Vázquez, R., Guerrero, M. A., et al. 2012, MNRAS, 423 , 3753

Reipurth, B., Hartigan, P., Heathcote, S., Morse, J. A., \& Bally, J. 1997, AJ, 114, 757

Reipurth, B., Heathcote, S., Morse, J., Hartigan, P., \& Bally, J. 2002, AJ, 123, 362

Rodríguez-González, A., Esquivel, A., Raga, A. C., et al. 2012, AJ, 143, 60

Ruiz, M. T., Peimbert, A., Peimbert, M., \& Esteban, C. 2003, ApJ, 595, 247

Ruiz, N., Guerrero, M. A., Chu, Y.-H., \& Gruendl, R. A. 2011, AJ, 142, 91

Sabbadin, F. 1976, A\&A, 52, 291

Sahai, R., \& Trauger, J. T. 1998, AJ, 116, 1357.

Schönberner, D., Jacob, R., Steffen, M., et al. 2005, A\&A, 431, 963

Turatto, M., Cappellaro, E., Ragazzoni, R., Benetti, S., \& Sabbadin, F. 2002, A\&A, 384, 1062

Vázquez, R. 2012, ApJ, 751, 116

Villaver, E., Manchado, A., \& García-Segura, G. 2002, ApJ, 581, 1204

Yuan, H.-B., Liu, X.-W., Péquignot, D., et al. 2011, MNRAS, 411, 1035

Walton, N. A., Reay, N. K., Pottasch, S. R., \& Atherton, P. D. 1986, New Insights in Astrophysics. Eight Years of UV Astronomy with IUE, 263, 497

Wesson, R., \& Liu, X.-W. 2004, MNRAS, 351, 1026 
Martín A. Guerrero et al.: Unveiling shocks in PNe

Table 1. Sample of PNe with HST WFPC2 or WFC3 H $\alpha$ and [O III] observations.

\begin{tabular}{|c|c|c|c|c|c|c|c|c|c|}
\hline$\overline{\mathrm{PN} G}$ & Common name & $\begin{array}{c}\mathrm{H} \alpha \\
\text { Exp. time } \\
\text { (s) }\end{array}$ & $\begin{array}{c}{[\mathrm{O} \text { III }]} \\
\text { Exp. time } \\
\quad \text { (s) }\end{array}$ & Proposal ID & PN G & Common name & $\begin{array}{c}\mathrm{H} \alpha \\
\text { Exp. time } \\
\text { (s) }\end{array}$ & $\begin{array}{c}{[\mathrm{O} \text { III] }} \\
\text { Exp. time } \\
\quad \text { (s) }\end{array}$ & Proposal ID \\
\hline $000.3+12.2$ & IC 4634 & 1000 & 1000 & 6856 & $084.2+01.0$ & K 4-55 & 2460 & 2440 & 11956 \\
\hline $001.2+02.1$ & Hen 2-262 & 280 & 280 & 9356 & $084.9-03.4$ & NGC $7027^{c}$ & 500 & 100 & 11122 \\
\hline $001.7-04.4$ & H 1-55 & 200 & 280 & 9356 & $089.8-05.1$ & IC 5117 & 240 & 320 & 8307 \\
\hline $002.3-03.4$ & Н 2-37 & 280 & 280 & 9356 & $096.4+29.9$ & NGC 6543 & 800 & 1600 & 5403 \\
\hline $002.4+05.8$ & NGC 6369 & 640 & 640 & 9582 & $106.5-17.6$ & NGC 7662 & 200 & 500 & $6117,6943,8390$ \\
\hline $002.7-04.8$ & M 1-42 & 900 & 1800 & 11185 & $111.8-02.8$ & $\mathrm{Hb} 12$ & 1600 & 1600 & 11093 \\
\hline $002.9-03.9$ & Н 2-39 & 280 & 280 & 9356 & $138.8+02.8$ & IC 289 & 2000 & 2000 & 11956 \\
\hline $003.5-04.6$ & NGC 6565 & 160 & 320 & 11122 & $144.1+06.1$ & NGC 1501 & 1600 & 2000 & 11956 \\
\hline $003.6+03.1$ & M 2-14 & 280 & 280 & 9356 & $189.1+19.8$ & NGC 2371-72 & 1600 & 1600 & 11093 \\
\hline $003.8+05.3$ & Н 2-15 & 280 & 280 & 9356 & $197.8+17.3$ & NGC 2392 & 400 & 400 & 8499 \\
\hline $003.9-03.1$ & KFL 7 & 280 & 280 & 9356 & $215.2-24.2$ & IC 418 & 888 & 360 & 6353,7501 \\
\hline $004.0-03.0$ & M 2-29 & 200 & 160 & 9356 & $231.8+04.1$ & NGC 2438 & 2080 & 2080 & 11827 \\
\hline $004.1-03.8$ & KFL 11 & 280 & 280 & 9356 & $215.6+03.6$ & NGC 2346 & 200 & 120 & 7129 \\
\hline $004.8-22.7$ & Hen 2-436 & 200 & 160 & 9356 & $234.8+02.4$ & NGC 2440 & 1600 & 1600 & 11090 \\
\hline $004.8+02.0$ & H 2-25 & 400 & 400 & 9356 & $249.0+06.9$ & SaSt 1-1 & 200 & 280 & 8332 \\
\hline $005.2-18.6$ & StWr 2-21 & 280 & 280 & 9356 & $261.0+32.0$ & NGC 3242 & 100 & 1200 & $6117,7501,8773$ \\
\hline $006.1+08.3$ & M 1-20 & 200 & 160 & 9356 & $261.9+08.5$ & NGC 2818 & 1600 & 2000 & 11956 \\
\hline $006.3+04.4$ & H 2-18 & 280 & 280 & 9356 & $272.1+12.3$ & NGC 3132 & 400 & 1200 & 6221,8390 \\
\hline $006.4+02.0$ & M 1-31 & 780 & 160 & 9356 & $285.6-02.7$ & Hen 2-47 & 1600 & 1600 & 11090 \\
\hline $006.8-19.8$ & Wray $16-423$ & 200 & 160 & 9356 & $285.7-14.9$ & IC 2448 & 200 & 320 & 11122 \\
\hline $006.8+04.1$ & M3-15 & 200 & 160 & 9356 & $294.6+04.7$ & NGC 3918 & 140 & 320 & 11122 \\
\hline $007.5+04.3$ & Th 4-1 & 280 & 280 & 9356 & $305.1+01.4$ & Hen 2-90 & 2325 & 1210 & 8345,9102 \\
\hline $008.2+06.8$ & Hen 2-260 & 200 & 460 & 9356 & $307.5-04.9$ & MyCn 18 & 600 & 1400 & 6221 \\
\hline $008.6-02.6$ & $\mathrm{MaC} 1-11$ & 280 & 280 & 9356 & $309.1-04.3$ & NGC 5315 & 1600 & 1600 & 11090 \\
\hline $009.3+05.7$ & Hen 3-1475 & 830 & 800 & 7285 & $312.3+10.5$ & NGC 5307 & 1600 & 1600 & 11090 \\
\hline $010.0+00.7$ & NGC 6537 & 1240 & 1000 & 6502 & $319.6+15.7$ & IC $4406^{d}$ & 540 & 600 & 8726,9314 \\
\hline $010.8+18.0$ & M 2-9 & 1240 & 1000 & 6502 & $324.0+03.5$ & PM 1-89 & 4900 & 2900 & 5404,5864 \\
\hline $010.8-01.8$ & NGC 6578 & 160 & 320 & 11122 & $327.8+10.8$ & NGC 5882 & 140 & 380 & 11122 \\
\hline $019.4-05.3$ & M 1-61 & 240 & 320 & 8307 & $331.1-05.7$ & PC 11 & 200 & 280 & 8332 \\
\hline $025.3+40.8$ & IC 4593 & 1600 & 1600 & 11093 & $331.3-12.1$ & Hen 3-1357 & 240 & 368 & 6039,8390 \\
\hline $025.8-17.9$ & NGC 6818 & 520 & 1300 & $6792,7501,8773$ & $331.7-01.0$ & $\mathrm{Mz} 3^{e}$ & 1260 & 1160 & 6856,9050 \\
\hline $027.6+04.2$ & M 2-43 & 520 & 1800 & 8307 & $341.8+05.4$ & NGC 6153 & 1000 & 1200 & 8594 \\
\hline $034.6+11.8$ & NGC 6572 & 180 & 840 & 7501,9839 & $349.5+01.0$ & NGC $6302^{a}$ & 2100 & 2220 & 11504 \\
\hline $036.1-57.1$ & NGC 7293 & 1800 & 1800 & 5977 & $351.1+04.8$ & M 1-19 & 160 & 160 & 9356 \\
\hline $037.7-34.5$ & NGC 7009 & 400 & 320 & 8114 & $351.9-01.9$ & Wray 16-286 & 200 & 280 & 9356 \\
\hline $037.8-06.3$ & NGC 6790 & 160 & 200 & 8307 & $352.6+03.0$ & H 1-8 & 200 & 280 & 9356 \\
\hline $043.1+37.7$ & NGC 6210 & 320 & 320 & 6792 & $353.5-05.0$ & $\mathrm{JaFu} 2^{f}$ & 3600 & 2000 & 6780 \\
\hline $054.1-12.1$ & NGC 6891 & 1280 & 320 & 11122 & $354.5+03.3$ & Th 3-4 & 280 & 280 & 9356 \\
\hline $054.2-03.4$ & - Necklace Nebula ${ }^{a}$ & 2000 & 2000 & 12675 & $354.9+03.5$ & Th 3-6 & 280 & 400 & 9356 \\
\hline $057.9-01.5$ & Hen $2-447$ & 520 & 1800 & 8307 & $355.4-02.4$ & M 3-14 & 200 & 160 & 9356 \\
\hline $060.1-07.7$ & NGC 6886 & 1120 & 1020 & $7501,8345,8773$ & $355.9+03.6$ & H 1-9 & 280 & 280 & 9356 \\
\hline $060.8-03.6$ & NGC 6853 & 2000 & 1000 & 8726 & $356.1-03.3$ & H 2-26 & 280 & 280 & 9356 \\
\hline $063.1+13.9$ & NGC 6720 & 480 & 720 & 7632,8726 & $356.5-03.6$ & Н 2-27 & 360 & 400 & 9356 \\
\hline $064.1+04.3$ & M 1-92 & 680 & 2080 & 6533 & $356.9+04.4$ & M3-38 & 280 & 280 & 9356 \\
\hline $064.7+05.0$ & $\mathrm{BD}+30^{\circ} 3639$ & 484 & 900 & 8116,8390 & $357.1-04.7$ & H 1-43 & 200 & 280 & 9356 \\
\hline $065.0-27.3$ & Ps $1^{b}$ & 11420 & 1040 & 6751 & $357.2+02.0$ & H 2-13 & 280 & 280 & 9356 \\
\hline $071.6-02.3$ & M3-35 & 520 & 1000 & 8307 & $358.5-04.2$ & H 1-46 & 160 & 160 & 9356 \\
\hline $073.0-02.4$ & K 3-76 & 6 & 18 & 6943 & $358.5+02.9$ & Wray $16-282$ & 280 & 280 & 9356 \\
\hline $074.5+02.1$ & NGC 6881 & 280 & 320 & 8307 & $358.9+03.4$ & H 1-19 & 200 & 280 & 9356 \\
\hline $082.1+07.0$ & NGC 6884 & 1100 & 560 & 8345,8390 & $359.2+04.7$ & Th 3-14 & 280 & 400 & 9356 \\
\hline $082.5+11.3$ & NGC 6833 & 40 & 3 & 6943,6353 & $359.3-00.9$ & $\mathrm{Hb} 5$ & 1300 & 1000 & 6502 \\
\hline $083.5+12.7$ & NGC 6826 & 100 & 100 & 6117 & & & & & \\
\hline
\end{tabular}

Notes. ${ }^{(a)}$ Source observed with the WFC3 camera. ${ }^{(b)}$ PN in the globular cluster NGC 7078. ${ }^{(c)}$ The nebula is mostly covered by the WFPC2PC1 chip, but no completely. ${ }^{(d)}$ The nebula is covered by two adjacent WFPC2 observations. ${ }^{(e)}$ Possible symbiotic star. ${ }^{(f)}$ PN in the globular cluster NGC 6441. 
Table 2. $[\mathrm{O}$ III $] / \mathrm{H} \alpha$ type and basic parameters of planetary nebulae.

\begin{tabular}{|c|c|c|c|c|c|c|c|c|c|c|c|}
\hline $\begin{array}{l}\text { Common } \\
\text { name }\end{array}$ & $\begin{array}{c}{[\mathrm{O} \text { III }] / \mathrm{H} \alpha} \\
\text { class }\end{array}$ & Shape & $(\mathrm{kK})$ & $\begin{array}{c}N_{\mathrm{e}} \\
\left(\mathrm{cm}^{-3}\right)\end{array}$ & $\left({ }^{\prime \prime}\right)$ & $\begin{array}{c}d \\
(\mathrm{kpc})\end{array}$ & $\begin{array}{c}R \\
(\mathrm{pc})\end{array}$ & {$[\mathrm{O} \mathrm{III}] / \mathrm{H} \beta$} & {$[\mathrm{N} \mathrm{II]}] / \mathrm{H} \beta$} & Comments & References \\
\hline IC 4593 & $\mathrm{AB}$ & MS & 40 & 3000 & 7.5 & 1.57 & 0.06 & 560 & 12 & PS outflows & 1,2 \\
\hline IC 4634 & A & $\mathrm{E}, \mathrm{MS}$ & 49 & 2700 & 4.2 & 2.8 & 0.06 & 999 & 11 & PS outflows & 3,4 \\
\hline NGC 3918 & $\mathrm{AB}$ & E, MS & 150 & 5500 & 9.0 & 1.84 & 0.08 & 1657 & 84 & PS outflows & 1,5 \\
\hline NGC 6210 & A & PS, MS & 60 & 5100 & 8.0 & 2.10 & 0.08 & 1084 & 8.3 & PS outflows & $1,2,6$ \\
\hline NGC 6543 & $\mathrm{AB}$ & E, MS & 48 & 4600 & 9.4 & 1.50 & 0.07 & 613 & 43 & PS outflows & 1,7 \\
\hline NGC 6572 & $\mathrm{AC}$ & MP, MS & 69 & 10000 & 7.0 & 1.86 & 0.06 & 1107 & 32 & Outflow & 1,8 \\
\hline NGC 7009 & $\mathrm{AB}$ & E, MS & 87 & 3900 & 12.5 & 1.45 & 0.09 & 1270 & 22 & FLIERs & 1,2 \\
\hline IC 289 & $\mathrm{B}$ & $\mathrm{E}, \mathrm{MS}$ & $\ldots$ & 700 & 15.0 & 2.19 & 0.16 & 460 & 5 & & 1,9 \\
\hline IC 2448 & B & E, MS & 65 & 1100 & 11.0 & 2.20 & 0.12 & 1173 & 1.0 & & 1,10 \\
\hline NGC 1501 & B & E & 135 & 950 & 27.0 & 0.72 & 0.09 & 1151 & 9.93 & & 1,11 \\
\hline NGC 2371-2 & B & E & 100 & 2500 & 28.0 & 0.50 & 0.07 & 901 & 160 & Ansae/Lobe & 12 \\
\hline NGC 3242 & B & E, MS & 89 & 2000 & 18.6 & 1.00 & 0.09 & 1520 & 14 & FLIERs & 1,13 \\
\hline NGC 5307 & B & $\mathrm{E}, \mathrm{MS}$ & $\ldots$ & 2500 & 7.1 & 3.0 & 0.10 & 1462 & 11.07 & PS outflows & 9,14 \\
\hline NGC 5882 & B & E, MS & 68 & 5900 & 7.0 & 1.70 & 0.06 & 1335 & 15.5 & & 1,15 \\
\hline NGC 6153 & B & E, MS & 109 & 5100 & 12.5 & 1.10 & 0.07 & 1189 & 64.2 & FLIERs & 1,16 \\
\hline NGC 6578 & B & E, MS & $\ldots$ & 4700 & 6.0 & 2.9 & 0.08 & 893 & 36 & & 1,9 \\
\hline NGC 6818 & B & E, MS & 160 & 2100 & 10.0 & 1.64 & 0.08 & 1190 & 46 & & 1,17 \\
\hline NGC 6826 & B & $\mathrm{E}, \mathrm{MS}$ & 50 & 3400 & 12.7 & 1.30 & 0.08 & 734 & 9.3 & FLIERs & 1,2 \\
\hline NGC 6884 & B & PS, MS & 95 & 2300 & 8.0 & 2.55 & 0.10 & 1966 & 26 & PS outflows & 1,18 \\
\hline NGC 6891 & B & $\mathrm{E}, \mathrm{MS}$ & 50 & 3000 & 6.6 & 2.9 & 0.09 & 868 & 12 & & $1,9,19$ \\
\hline NGC 7662 & B & E, MS & 111 & 2700 & 14.8 & 1.19 & 0.08 & 1223 & 2 & FLIERs & 1,20 \\
\hline $\mathrm{BD}+30^{\circ} 3639$ & $\mathrm{C}$ & $\mathrm{E}, \mathrm{MS}$ & 32 & 40800 & 3.0 & 1.3 & 0.02 & 9 & $\ldots$ & Collimated outflows & 1,9 \\
\hline Н $1-9$ & $\mathrm{C}$ & E & 35 & 13000 & 0.5 & 4.4 & 0.01 & 153.1 & 89.9 & & 21 \\
\hline H $1-46$ & $\mathrm{C}$ & PS & 41 & & 0.7 & 3.5 & 0.01 & 540 & 149 & Possible PS outflows & 22 \\
\hline $\mathrm{Hb} 12$ & $\mathrm{C}$ & B & 35 & 500000 & 5.5 & 10.0 & 0.27 & 509.3 & 22.1 & & 23,24 \\
\hline Hen 2-47 & $\mathrm{C}$ & & $\ldots$ & $\ldots$ & 5.5 & 5.5 & 0.15 & 12 & 458 & & 9 \\
\hline Hen 2-260 & $\mathrm{C}$ & $\mathrm{E}$ & 27 & $\ldots$ & 0.5 & 6.9 & 0.02 & $\ldots$ & 213 & Ansae & 9,22 \\
\hline Hen 2-447 & $\mathrm{C}$ & MP & $\ldots$ & $\ldots$ & 1.3 & 7.6 & 0.05 & 427 & 708 & & 9,23 \\
\hline Hen 3-1357 & $\mathrm{C}$ & B & $\ldots$ & $\ldots$ & 1.5 & 5.6 & 0.04 & $\ldots$ & $\ldots$ & Possible PS outflows & - \\
\hline IC 418 & $\mathrm{C}$ & E, MS & 38 & 20000 & 6.2 & 1.2 & 0.04 & 85.9 & 206.8 & & 1,25 \\
\hline IC 5117 & $\mathrm{C}$ & MP & 57 & 38900 & 0.7 & 1.3 & 0.005 & 1346.4 & 47.9 & & 26 \\
\hline M 1-19 & $\mathrm{C}$ & E & $\ldots$ & $\ldots$ & 1.3 & 5.5 & 0.03 & 546 & 214 & Low $\mathrm{S} / \mathrm{N}$ & 22 \\
\hline M 1-20 & $\mathrm{C}$ & E, MS & 81 & 11200 & 0.8 & 3.4 & 0.01 & 830 & 78 & & 1,22 \\
\hline M 1-31 & $\mathrm{C}$ & MP & 90 & $\ldots$ & 3.5 & 4.2 & 0.07 & 737 & 516 & Possible PS outflows & 22 \\
\hline M 1-42 & $\mathrm{C}$ & $\mathrm{E}$ & $\ldots$ & $\ldots$ & 7.8 & $\ldots$ & & 590 & 246 & Ansae/bipolar lobes & 22 \\
\hline M 1-61 & $\mathrm{C}$ & MP & 73 & $\ldots$ & 1.8 & 2.4 & 0.02 & 914 & 170 & Possible PS outflows & 9 \\
\hline M 2-43 & $\mathrm{C}$ & $\mathrm{E}$ & 57 & $\ldots$ & 0.6 & 5.0 & 0.01 & 629 & 1162 & Ansae & 9,23 \\
\hline MyCn 18 & $\mathrm{C}$ & B & 51 & $\ldots$ & 7.2 & 3.1 & 0.11 & 294 & $\ldots$ & PS outflows & - \\
\hline NGC 2346 & $\mathrm{C}$ & B & 112 & 440 & 27.3 & 0.9 & 0.12 & 1001 & 395 & & 1,27 \\
\hline NGC 6565 & $\mathrm{C}$ & E & 120 & 3200 & 5.0 & 2.25 & 0.06 & $\ldots$ & $\ldots$ & Ansae & 1,28 \\
\hline NGC 6720 & $\mathrm{C}$ & E, MS & 116 & 800 & 35.0 & 0.7 & 0.12 & 1064 & 297 & & 1,9 \\
\hline NGC 6790 & $\mathrm{C}$ & E, MS & 74 & 9600 & 2.5 & 1.5 & 0.02 & 1274 & 71 & Bipolar lobes & 9 \\
\hline NGC 6881 & $\mathrm{C}$ & Q & 45 & 7300 & 2.5 & 2.5 & 0.03 & 1851 & 603 & $\mathrm{H}_{2}$ hourglass lobes & 9 \\
\hline NGC 6886 & $\mathrm{C}$ & $\mathrm{B}$ & 129 & 4900 & 3.8 & 5.3 & 0.10 & $\ldots$ & 346 & & 1,9 \\
\hline NGC 7027 & $\mathrm{C}$ & $\mathrm{B}$ & 175 & 30200 & 10.0 & 0.9 & 0.04 & $\ldots$ & $\ldots$ & PS outflows & 1 \\
\hline H $1-8$ & $\mathrm{D}$ & MP & $\ldots$ & $\ldots$ & 1.1 & 5.3 & 0.03 & 993 & 2177 & Low S/N & 22 \\
\hline Н $2-13$ & D & $\mathrm{E}, \mathrm{MS}$ & $\ldots$ & $\ldots$ & 1.6 & $\ldots$ & & 2081 & 529 & Low $\mathrm{S} / \mathrm{N}$ & 22 \\
\hline H 2-18 & $\mathrm{D}$ & B & $\ldots$ & $\ldots$ & 1.9 & 7.8 & 0.07 & 1427 & 30 & Low $\mathrm{S} / \mathrm{N}$ & 22 \\
\hline H $2-27$ & D & B & $\ldots$ & $\ldots$ & 1.8 & $\ldots$ & $\ldots$ & 816 & 887 & Low $\mathrm{S} / \mathrm{N}$ & 22 \\
\hline Н 2-37 & D & PS & $\ldots$ & $\ldots$ & 2.0 & $\ldots$ & $\ldots$ & 811 & 198 & Low $\mathrm{S} / \mathrm{N}$ & 22 \\
\hline Н 2-39 & $\mathrm{D}$ & $\ldots$ & $\ldots$ & $\ldots$ & 2.2 & $\ldots$ & $\ldots$ & 1509 & $\ldots$ & Low $\mathrm{S} / \mathrm{N}$ & 22 \\
\hline Hen 2-90 & $\mathrm{D}$ & PS & $\ldots$ & $\ldots$ & 2.3 & $\ldots$ & $\ldots$ & 205 & 141 & Fast collimated outflows & 9 \\
\hline Hen 3-1475 & $\mathrm{D}$ & PS & $\ldots$ & $\ldots$ & 5.8 & $\ldots$ & $\ldots$ & $\ldots$ & $\ldots$ & Fast collimated outflows & - \\
\hline KFL 11 & $\mathrm{D}$ & $\ldots$ & $\ldots$ & $\ldots$ & 1.0 & $\ldots$ & $\ldots$ & 910 & 127 & Low $\mathrm{S} / \mathrm{N}$ & 22 \\
\hline M 1-92 & $\mathrm{D}$ & $\mathrm{B}$ & $\ldots$ & $\ldots$ & 5.2 & $\ldots$ & $\ldots$ & $\ldots$ & $\ldots$ & & - \\
\hline M 2-9 & $\mathrm{D}$ & Q & 35 & 3200 & 18.5 & 1.7 & 0.15 & 90 & 460 & & 9 \\
\hline M 2-29 & $\mathrm{D}$ & E, MS & 68 & $\ldots$ & 2.0 & 8.6 & 0.08 & 516 & 34 & Low $\mathrm{S} / \mathrm{N}$ & 22 \\
\hline M3-14 & $\mathrm{D}$ & B & $\ldots$ & $\ldots$ & 1.9 & 5.7 & 0.05 & 1065 & 1196 & Low $\mathrm{S} / \mathrm{N}$ & 22 \\
\hline M3-15 & D & B & $\ldots$ & 4200 & 2.0 & 5.3 & 0.05 & 1064 & 224 & Low S/N & 22 \\
\hline M3-35 & $\mathrm{D}$ & MP & $\ldots$ & $\ldots$ & 1.0 & 1.8 & 0.01 & 1123 & 34 & & 9 \\
\hline M 3-38 & $\mathrm{D}$ & B & 153 & $\ldots$ & 0.7 & 5.9 & 0.02 & 1939 & 598 & Low $\mathrm{S} / \mathrm{N}$, probable $\mathrm{Q}$ & 9,22 \\
\hline $\mathrm{MaC} 1-11$ & $\mathrm{D}$ & $\ldots$ & $\ldots$ & $\ldots$ & 1.4 & $\ldots$ & $\ldots$ & 995 & 49 & Low $\mathrm{S} / \mathrm{N}$ & 22 \\
\hline Necklace Nebula & $\mathrm{D}$ & & $\ldots$ & 820 & 13.5 & 4.6 & 0.30 & 534.6 & 12.7 & & 29 \\
\hline NGC 2392 & $\mathrm{D}$ & E, MS & 47 & 900 & 23.0 & 1.28 & 0.14 & 1406 & 160 & & 1,9 \\
\hline NGC 5315 & $\mathrm{D}$ & & & 10200 & 5.4 & 2.62 & 0.07 & 840 & 266 & & 1,9 \\
\hline NGC 6369 & D & $\mathrm{E}, \mathrm{MS}$ & 66 & 3800 & 16.0 & 1.55 & 0.12 & 1484 & 183 & & 1,9 \\
\hline PM 1-89 & $\mathrm{D}$ & E & $\ldots$ & $\ldots$ & 3.7 & $\ldots$ & $\ldots$ & $\ldots$ & $\ldots$ & Low $\mathrm{S} / \mathrm{N}$ & - \\
\hline Ps 1 & $\mathrm{D}$ & E, MS & $\ldots$ & $\ldots$ & 1.2 & 8.4 & 0.05 & 271 & 4 & & 9 \\
\hline StWr 2-21 & D & E & $\ldots$ & $\ldots$ & 0.9 & $\ldots$ & $\ldots$ & 1065 & 8 & Low S/N & 9 \\
\hline Th 3-4 & $\mathrm{D}$ & $\mathrm{B}$ & $\ldots$ & $\ldots$ & 0.9 & $\ldots$ & $\ldots$ & 2005 & 821 & Low $\mathrm{S} / \mathrm{N}$ & 22 \\
\hline Wray $16-286$ & $\mathrm{D}$ & Q & $\ldots$ & $\ldots$ & 1.1 & $\ldots$ & $\ldots$ & 1259 & 419 & Low S/N, quadrupolar & 9 \\
\hline Wray $16-423$ & D & $\ldots$ & $\ldots$ & $\ldots$ & 0.8 & $\ldots$ & $\ldots$ & 1198 & 19 & & 9 \\
\hline
\end{tabular}

References. (1) Frew (2008); (2) Kwitter \& Henry (1998); (3) Guerrero et al. (2008); (4) Hyung et al. (1999); (5) Ercolano et al. (2003); (6) Pottasch et al. (2009); (7) Wesson \& Liu (2004); (8) Hyung et al. (1994b); (9) Acker et al. (1992); (10) Guiles et al. (2007); (11) Ercolano et al. (2004); (12) Pottasch et al. (1981); (13) Ruiz et al. (2011); (14) Ruiz et al. (2003); (15) Pottasch et al. (2004); (16) Yuan et al. (2011). (17) Pottasch et al. (2005); (18) Hyung \& Aller (1997); (19) Méndez et al. (1988); (20) Hyung et al. (1997); (21) Cavichia et al. (2010); (22) Acker et al. (1991); (23) Phillips (2004); (24) Hyung \& Aller (1996); (25) Hyung et al. (1994b); (26) Hyung et al. (2001); (27) Sabbadin (1976); (28) Turatto et al. (2002); (29) Corradi et al. (2011). 